\title{
Bodypart Localization for Pressure Ulcer Prevention
}

\author{
Jason J. Liu ${ }^{1}$, Ming-Chun Huang ${ }^{1}$, Wenyao $\mathrm{Xu}^{2}$, Majid Sarrafzadeh ${ }^{1}$
}

\begin{abstract}
Pressure ulcers, commonly called bedsores, are defined as injuries to the skin and underlying tissue resulting from prolonged pressure usually on bony areas of the body, such as heels and hips. In order to alleviate pressure accumulation at high-risk regions, the current practice in clinics requires caregivers to reposition their patients every two hours. This relies on the engagement of nursing services and places large burdens on the caregivers. In this study, a bedsore monitoring method using Pictorial Structure models is introduced to localize pressure distributions of the body. A pilot study including 12 subjects reveals that the proposed method enables reliable localization of bodyparts with $\mathbf{8 9 . 8 \%}$ accuracy in common lying postures.
\end{abstract}

\section{INTRODUCTION AND RELATED WORK}

One of the main problems for post-surgical patients and elderly patients is formation of pressure ulcers [1]. Pressure ulcers, or bedsores, are localized injury to body tissue, usually near the bone, resulting from lack of movement and obstructed blood circulation. In hospital settings, caregivers need to be attentive to subjects that are more susceptible to this condition, and take action to relieve pressure on the highly sensitive locations by changing their sleep postures. Current best practices in nursing involve turning patients every several hours [2]. Higher quality mattresses or softer overlays that attempt to distribute pressure more evenly have been used, while dynamic surfaces such as alternating pressure overlays that employ circulating air or liquid have shown to be effective [3].

Given that pressure ulcers can develop very quickly, the autonomous monitoring of high risk areas is a realistic goal. The goal of any medical system that prevents the formation of pressure ulcers requires the analysis of sleeping postures, as well as notifications of susceptible and impending pressure points on the patient's body. It is well known which bodyparts are most prone to pressure ulcers: sacrum, heels, trochanter (hips), and elbows [4]. Therefore, there is indeed a need to automatically locate these bodyparts, measure the accumulated pressure, and notify hospital staff of impending issues.

With recent advances in technology, pressure sensors are being increasingly deployed in various devices for measuring pressure on the body. Current commercial systems in the form of sheets are undergoing greater use [5], [6]. These sensors have a variety of resolutions and are able to accurately measure pressure distributions under the body. Other research projects are more focused on developing low-cost

\footnotetext{
${ }^{1}$ Department of Computer Science, University of California, Los Angeles

${ }^{2}$ Department of Computer Science and Engineering, the State University of New York (SUNY) at Buffalo, New York

E-mail: \{jasonliu,mingchuh,majid\}@cs.ucla.edu, wenyaoxu@buffalo.edu
}

disposable sheets compared to existing commercial systems. A capacitive pressure sensor array was designed by Yip et al. with an estimated low cost of $\$ 50 / \mathrm{m}^{2}$ [7]. Our previous work involved designing an eTextile bedsheet that employed the piezoelectric characteristics of such materials [8].

Using a commerical pressure sensitive mat, Farshbaf et al. located bodyparts with a reported average accuracy of $85.7 \%$ [9]. Their method involved extracting the body boundary through filtering, dilation, and edge detection. Then, with Constrained Delaunay Triangulation, they were able to prune a skeletal graph. Finally, by applying Dynamic Time Warping to the skeleton, the nodes and leaves can be matched to a predefined bodypart template of the human body. In conjunction with Siemens magnetic resonance imaging (MRI), Grimm et al. used constrained articulated ellipsoid modelling of the body to minimize the error between the pressure image and generated images [10]. In this case, bodypart localization helped to fine tune specific scans of the patient. To our knowledge, these works are the only published prior art in bodypart localization using pressure sensors with the purpose of pressure ulcer prevention.

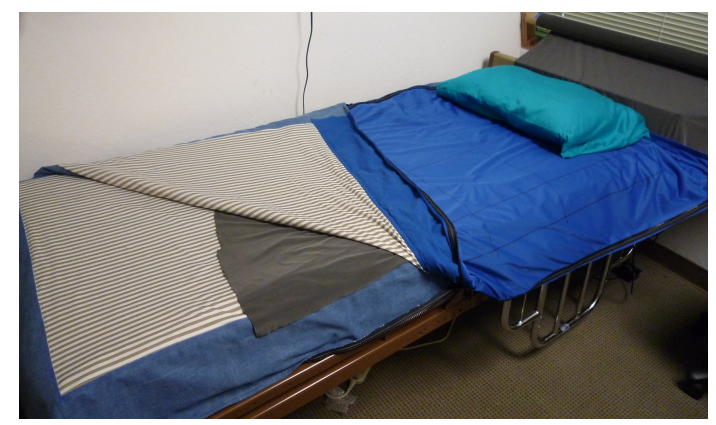

Fig. 1. The pressure sensitive bedsheet setup with sensing eTextile layers.

In this paper, we focus on localizing bodyparts within pressure images. We employ a dense pressure sensitive textile bedsheet and apply pressure image analysis for bodypart localization. In contrast to the previously mentioned work that reduced a pressure image of the body to a skeletonized frame, our method uses a Pictorial Structure model of the body [11] and calculates the best locations based on both the appearance and the spatial information of the pressure image.

With the end goal of monitoring pressure ulcer occurrence, the locations of bodyparts can be found and this information can be fed into patient monitoring systems that track the accumulation of pressure and generate alerts using efficient turning schedules [12], or risk maps [13]. The work presented in this paper targets the accurate localization of bodyparts. 


\section{Pressure Sensor}

Figure 1 shows the prototype of the bedsheet system. The sensor system consists of three components: a $64 \times 128$ pressure sensor array, a data sampling unit, and a tablet for data analysis and storage. The sensor array is based on eTextile material which is fiber-based yarn coated with piezoelectric polymer [14]. The initial resistance of the eTextile material is high. As external force is applied to the surfaces of the material, the eTextile fibers are squeezed together and, due to its pressure sensitive characteristics, the electrical resistance decreases in that region.

The bedsheet has a three-layer sandwiched structure. The top layer is regular fabric that is coated with 64 parallel conductive lines. The middle layer is the eTextile material and the bottom layer has 128 conductive lines arranged perpendicular to the top 64 lines. At each intersection of conductive lines, the structure forms a pressure sensitive resistor. There are effectively 8192 pressure sensors in total.

\section{METHOD}

This method is based on the pictorial structures model, proposed by Fischler and Elschlager [11], for identifying structured objects in images. Felzenszwalb and Huttenlocher improved the matching efficiency of the parts based algorithm using distance transforms within dynamic programming [15]. The original formulation of finding bodyparts is described here.

Each part of the human body can be represented as a vertex in a tree. For instance, the head, shoulders, hands and feet are nodes in a graph connected by edges. Given an image $I$, we define a mismatch cost $m_{j}\left(I, l_{j}\right)$ as a measure of how much the image $I$ matches a bodypart $j$ at the location $l_{j}=$ $\left(x_{j}, y_{j}, \theta_{j}\right)$ where $x_{j}, y_{j}$ are the co-ordinates of the center of the bodypart model with rotation angle $\theta_{j}$. A low value of mismatch means the bodypart is well recognized at that location.

In addition to the cost for how well the appearance of each bodypart matches its template, there is a cost for the relationship between bodypart locations. For bodyparts $i$ and $j$, a deformation cost $d_{i j}\left(l_{i}, l_{j}\right)$ gives a measure of how far the bodyparts deviate from their expected respective locations. For instance, the hip and shoulders are known to be separated by the spine length. So for a given set of bodypart templates and connections between them, the best configuration of bodyparts $L=\left(l_{1}, \ldots, l_{n}\right)$ in an image minimizes the total cost of mismatch for all parts and deformation between all pairs of parts:

$$
\arg \min _{L}\left(\sum_{\text {allparts }} m_{j}\left(I, l_{j}\right)+\sum_{\text {allpairs }} d_{i j}\left(l_{i}, l_{j}\right)\right) .
$$

This formulation takes exponential time in the number of bodyparts $n$, however with the tree representation of bodyparts, this can be solved more efficiently as a chain of computations using dynamic programming as follows. For any leaf vertex (i.e. has no children), its best location $\hat{l}_{j}$ can be calculated as a function of its parent location $l_{i}$. So the best leaf vertex location is a function of parent location:

$$
B_{j}\left(l_{i}\right)=\arg \min _{l_{j}}\left(m_{j}\left(I, l_{j}\right)+d_{i j}\left(l_{i}, l_{j}\right)\right) .
$$

For a non-leaf, non-root vertex, its best location $\hat{l}_{j}$ as a function of its parent location $l_{i}$ is given by

$B_{j}\left(l_{i}\right)=\arg \min _{l_{j}}\left(m_{j}\left(I, l_{j}\right)+d_{i j}\left(l_{i}, l_{j}\right)+\sum_{C h(j)} B_{c}\left(l_{j}\right)\right)$,

where $C h(j)$ means children vertices of $j$ and $B_{c}\left(l_{j}\right)$ are the costs of the best locations of the children of $j$. This is already calculated and memoized via the dynamic programming methodology.

Then for the root vertex (no parent), its best location is

$$
\hat{l}_{j}=\arg \min _{l_{j}}\left(m_{j}\left(I, l_{j}\right)+\sum_{C h(j)} B_{c}\left(l_{j}\right)\right),
$$

where $B_{c}\left(l_{j}\right)$ is known for each of the children of $j$. This formulation (Equations 2 to 4 ) reduces the number of computations from exponential to polynomial while still producing the globally optimal solution.

To compute the mismatch cost $m_{j}\left(I, l_{j}\right)$ for each bodypart, we select a representative template and, in this paper, the bodyparts are simply represented as rectangular boxes surrounded by a border due to ease of calculation (Figure 2). The size of template is proportional to the height of the subject. To account for different pressure images due to varying weights of the subjects, the image $I$ is binarized at different thresholds. A convolution operation of this kernel with the binary images efficiently gives the mismatch cost at all locations. This computes how many pixels do not match inside the inner box and within the border. Then, mismatch cost is the smallest cost across all threshold levels for each location.

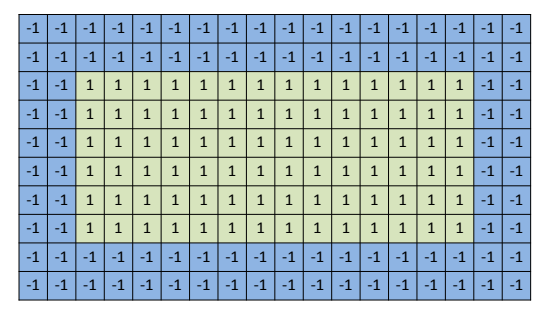

Fig. 2. Template for bodypart mismatch cost. The mismatch cost of the bodypart model is the sum of mismatched pixels within the inner rectangle and matching pixels in the border region.

To compute the deformation cost $d_{i j}\left(l_{i}, l_{j}\right)$ between bodyparts, we define this cost to have the form

$$
d_{i j}\left(l_{i}, l_{j}\right)=\left\|T_{i j}\left(l_{i}\right)-T_{j i}\left(l_{j}\right)\right\| .
$$


This is a simple distance metric between locations transformed to a common space. The transforms can be translations, rotations, and scaling, hence they are invertible (See Fig. 3). The effect of the transforms is to specify the expected relations between the locations, i.e. if two bodyparts have no deformation cost and are correctly positioned, then the transformed locations will coincide. The pairwise distance metric takes quadratic time in the number of locations. For every location of the parent, we want to compute the distance to each location of the child. On the surface, quadratic time may seem satisfactory but this can be improved to linear time through the use of distance transforms [16]. In fact, every pairwise distance between $l_{i}$ and $l_{j}$ need not be computed. From Equation 2, only the smallest sum of deformation cost and mismatch cost is needed at each location of $l_{i}$.

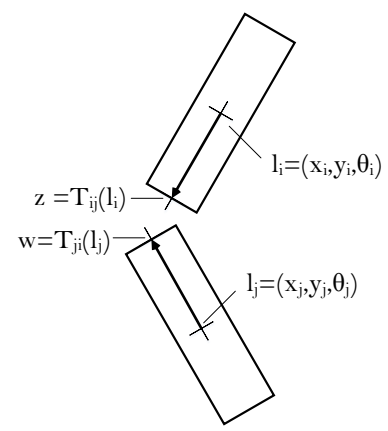

Fig. 3. Example transformation of bodypart models

The generalized form of a distance transform given a function $f$ is

$$
D(z)=\min _{w}(\|z-w\|+f(w))
$$

where $z$ and $w$ are locations in a grid. This formulation states that, for every location $z$, it finds the closest location $w$ with the smallest value for $f(w)$.

In Equation 6, we can map $z$ to a translated location for $l_{i}$, i.e. $z=T_{i j}\left(l_{i}\right)$. $w$ maps to a translated location for $l_{j}$, i.e. $w=T_{j i}\left(l_{j}\right) . f(w)$ is a function that maps to $f(w)=$ $m_{j}\left(I, T_{j i}{ }^{1}\left(l_{j}\right)\right)$. So now Equations 2 and 3 become

$$
B_{j}\left(l_{i}\right)=D\left(T_{i j}\left(l_{i}\right)\right) .
$$

Now the final discussion describes how to speed up the calculation of Equation 7. For the leaf nodes, we initialize an array $D[x, y, \theta]$ with the values of $m_{j}\left(I, T_{j i}{ }^{1}\left(T_{j i}\left(l_{j}\right)\right)\right)$ which is just the mismatch cost of the child but translated to a new location in the same space as the parent. For non-leaf nodes, we initialize the array $D[x, y, \theta]$ with the values of

$$
m_{j}\left(I, T_{j i}^{1}\left(T_{i j}\left(l_{i}\right)\right)\right)+\sum_{C h(j)} B_{c}\left(T_{j i}^{1}\left(T_{i j}\left(l_{i}\right)\right)\right),
$$

which is the mismatch cost of the child (translated to a new location in the same space as the parent) plus the cost of the children. The values of $D[x, y, \theta]$ can be updated according to

$$
\begin{aligned}
& D[x, y, \theta]=\min (D[x, y, \theta], \\
& D[x-1, y, \theta]+k_{x}, \\
& D[x, y-1, \theta]+k_{y}, \\
&\left.D[x, y, \theta-1]+k_{\theta}\right),
\end{aligned}
$$

where $k_{x}, k_{y}, k_{\theta}$ are constants that account for different pixel scales. This is executed in sequence starting from array point $[1,1,1]$ linearly through the array which is followed by a second sweep in the reverse direction.
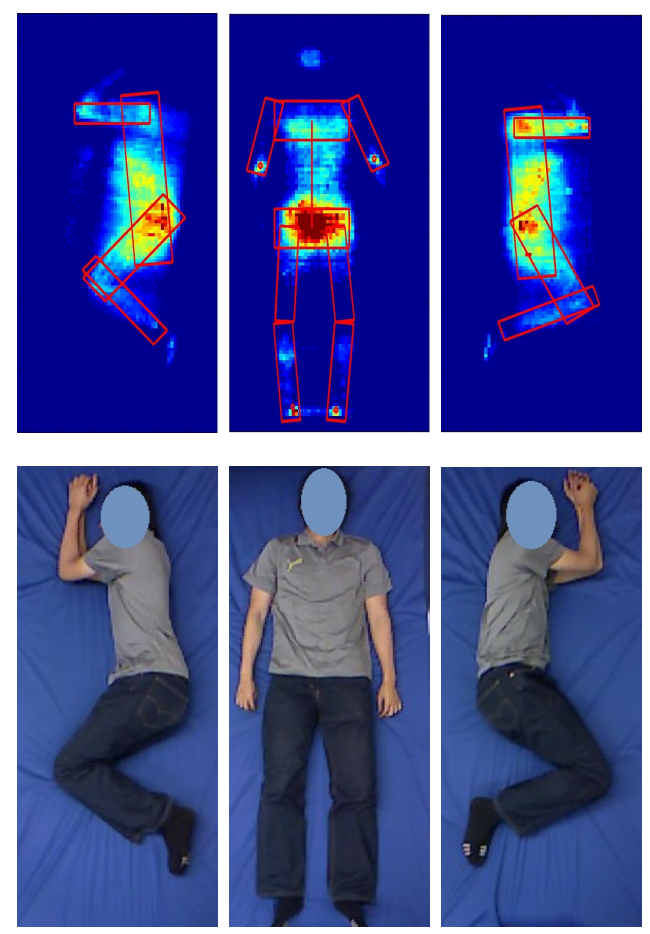

Fig. 4. Sample image results for bodypart localization in the right lateral, supine, and left lateral postures.

\section{EXPERIMENTAL RESULTS}

\section{A. Experimental Setup}

We run a pilot study to evaluate the performance of the system for localization of bodyparts. There are 12 subjects in the experiment with 9 male and 3 female subjects. The weights of the subjects range from $50 \mathrm{~kg}$ to $85 \mathrm{~kg}$, and height between $155 \mathrm{~cm}$ and $188 \mathrm{~cm}$. For the sake of consistency, the bedsheet system is also deployed on a standard twin-size coil spring mattress during the experiments.

In the data collection, 40 samples were recorded for each of the 3 postures (supine, left and right) for each subject. At fixed intervals, the pressure image of the subject's posture was recorded while the subject maintained a comfortable sleeping position. Variations in body, arm and leg positions were allowed and the system is tested on a range of positions that fall within the 3 defined postures. The following experiments were tested against hand labelled ground truth from direct overhead camera recordings. 


\section{B. Experimental Results}

Once each of the bodypart models is located, fine tuning of the locations of the high pressure points proceeds by finding the center of mass within the bodypart model. For the heels and elbow, the center of mass is restricted to the lower half of the bodypart model.

Figure 4 shows a sample of bodypart localization results in right lateral, supine, and left lateral postures. Lateral postures are defined by 4 bodypart models and supine posture is defined by 8 bodyparts models. Table I summarizes the accuracy of bodypart localization for the 3 postures compared against prior work by Farshbaf et al. [9] and Grimm et al. [10]. We use the same metrics as described in the prior work, where error is measured by Euclidean distance in inches between the tested and ground truth locations, and accuracy is calculated as the percentage of bodyparts that have error of less than 1 inch. In these experiments, the elbow resulted in the lowest localization accuracy. This can be explained by the relative weakness in pressure compared to the high pressure of the shoulder.

TABLE I

ACCURACY OF BODYPART LOCALIZATION

\begin{tabular}{|c|c|c|c|c|c|}
\hline Posture & Bodypart & $\begin{array}{c}\text { Accuracy } \\
\text { Farshbaf } \\
{[9]}\end{array}$ & $\begin{array}{c}\text { Error } \\
\text { Grimm } \\
{[10]}\end{array}$ & $\begin{array}{c}\text { Accuracy }{ }^{\dagger} \\
(\%)\end{array}$ & $\begin{array}{l}\text { Error } \\
\text { (inch) }\end{array}$ \\
\hline \multirow[t]{6}{*}{ Supine } & Shoulder & $93.3 \%$ & $1.7 " *$ & $92.4 \%$ & $1.2 "$ \\
\hline & Left Elbow & $86.7 \%$ & - & $87.1 \%$ & $1.6 "$ \\
\hline & Right Elbow & $80.0 \%$ & - & $86.2 \%$ & 1.7" \\
\hline & Sacrum & $93.3 \%$ & 1.7" & $94.7 \%$ & $0.9 "$ \\
\hline & Left Heel & $80.0 \%$ & - & $93.1 \%$ & $1.0 "$ \\
\hline & Right Heel & $80.0 \%$ & - & $93.7 \%$ & $1.0 "$ \\
\hline \multirow[t]{4}{*}{ Left } & Shoulder & $86.7 \%$ & $2.9 "$ & $94.5 \%$ & $0.9 ”$ \\
\hline & Elbow & $76.7 \%$ & - & $82.1 \%$ & $2.2^{\prime \prime}$ \\
\hline & Hip & $93.3 \%$ & $2.0 "$ & $91.9 \%$ & $1.3 "$ \\
\hline & Knee & - & $3.3 "$ & $90.0 \%$ & $1.8^{\prime \prime}$ \\
\hline \multirow[t]{4}{*}{ Right } & Shoulder & $90.0 \%$ & $2.3 "$ & $92.1 \%$ & $1.2^{\prime \prime}$ \\
\hline & Elbow & $96.7 \%$ & - & $84.5 \%$ & $2.0^{\prime \prime}$ \\
\hline & Hip & $90.0 \%$ & $2.0^{\prime \prime}$ & $92.2 \%$ & $1.2^{\prime \prime}$ \\
\hline & Knee & - & $3.3 "$ & $85.2 \%$ & $2.0 "$ \\
\hline Average & & $85.7 \%$ & $2.7 "$ & $89.8 \%$ & 1.4" \\
\hline
\end{tabular}

$\dagger$ Accuracy is percentage of bodyparts within 1 inch of groundtruth. $\ddagger$ Error is Euclidean distance from groundtruth location.

*Grimm et al. reported neck location instead.

Table II shows the average pressure of a sample subject. For this method of bodypart localization, the total accumulation of pressure can be recorded to facilitate pressure ulcer monitoring and prevention.

\section{CONCLusion}

This work presents a method to localize bodyparts using a pressure sensitive bedsheet. The purpose of such a system is to enable caregivers the ability to automatically identify where patients are at risk of developing pressure ulcers. In our experiments, we showed an overall localization accuracy of $89.8 \%$ for high risk regions such as the hip, heels, and shoulders. Future work also involves the ability to monitor accumulated pressure over continuous periods.
TABLE II

Average Pressure at High Risk Regions For a SubJect

\begin{tabular}{|l|c|}
\hline Bodypart (posture) & average pressure* $\left({\left.\mathrm{x} 10^{-3}\right)}^{-3}\right.$ ( $2.09 \pm 0.13$ \\
Sacrum (supine) & $2.01 \pm 0.13$ \\
Shoulder (lateral) & $1.92 \pm 0.11$ \\
Hip (lateral) & $1.52 \pm 0.12$ \\
Heel (supine) & $1.43 \pm 0.10$ \\
Elbow (supine) & $0.80 \pm 0.09$ \\
Shoulder (supine) & $<0.2$ \\
Non risk areas & $<$ \\
\hline
\end{tabular}

*Pressure values given are the pixel values

when sum of pixels is normalized to 1 .

\section{ACKNOWLEDGMENT}

The authors would like to thank Medisens Wireless Inc. for their contributions in discussion and building the hardware.

\section{REFERENCES}

[1] T. Perneger, C. Heliot, A.-C. Rae, and J.-M. Gaspoz, "Hospitalacquired pressure ulcers: Risk factors and use of preventive devices," Archives Internal Med., vol. 158, no. 17, pp. 1940-45, 1998.

[2] J. K. Stechmiller, L. Cowan, J. D. Whitney, L. Phillips, R. Aslam, A. Barbul, F. Gottrup, L. Gould, M. C. Robson, G. Rodeheaver et al., "Guidelines for the prevention of pressure ulcers," Wound Repair and Regeneration, vol. 16, no. 2, pp. 151-168, 2008.

[3] C. Chai and D. Bader, "The physiological response of skin tissues to alternating support pressures in able-bodied subjects," Journal of the Mechanical Behavior of Biomedical Materials, vol. 28, pp. 427-435, 2013.

[4] A. Thoroddsen, "Pressure sore prevalence: a national survey," Journal of Clinical Nursing, vol. 8, no. 2, pp. 170-179, 1999.

[5] Xsensor, http://www.xsensor.com.

[6] VistaMedical, http://www.pressuremapping.com.

[7] M. Yip, D. He, E. Winokur, A. G. Balderrama, R. Sheridan, and H. Ma, "A flexible pressure monitoring system for pressure ulcer prevention," in International Conference of IEEE Engineering in Medicine and Biology Society, EMBC. IEEE, 2009, pp. 1212-5.

[8] J. J. Liu, W. Xu, M.-C. Huang, N. Alshurafa, M. Sarrafzadeh, N. Raut, and B. Yadegar, "A dense pressure sensitive bedsheet design for unobtrusive sleep posture monitoring," in Pervasive Computing and Communications (PerCom), International Conference. IEEE, 2013, pp. 207-215.

[9] M. Farshbaf, R. Yousefi, M. Baran Pouyan, S. Ostadabbas, M. Nourani, and M. Pompeo, "Detecting high-risk regions for pressure ulcer risk assessment," in International Conference on Bioinformatics and Biomedicine. IEEE, 2013.

[10] R. Grimm, J. Sukkau, J. Hornegger, and G. Greiner, "Automatic patient pose estimation using pressure sensing mattresses," in Bildverarbeitung fur die Medizin 2011, ser. Informatik aktuell, 2011.

[11] M. A. Fischler and R. A. Elschlager, "The representation and matching of pictorial structures," IEEE Transactions on Computers, vol. 22, no. 1 , pp. 67-92, 1973.

[12] S. Ostadabbas, R. Yousefi, M. Faezipour, M. Nourani, and M. Pompeo, "Pressure ulcer prevention: An efficient turning schedule for bedbound patients," in Life Science Systems and Applications Workshop (LiSSA), 2011 IEEE/NIH. IEEE, 2011, pp. 159-162.

[13] I. Main, R. Miller, T. Russel, and M. Ousdahl, "Risk modeling for pressure ulcer formation," Aug. 4 2011, wO Patent App. PCT/CA2011/000,098. [Online]. Available: https://www.google.com/patents/WO2011091517A1?cl=en

[14] W. Xu, Z. Li, M.-C. Huang, N. Amini, and M. Sarrafzadeh, "eCushion: An eTextile Device for Sitting Posture Monitoring," in Body Sensor Networks (BSN), May 2011, pp. 194-199.

[15] P. F. Felzenszwalb and D. P. Huttenlocher, "Pictorial structures for object recognition," International Journal of Computer Vision, vol. 61, no. 1, pp. 55-79, 2005.

[16] G. Borgefors, "Distance transformations in digital images," Computer vision, graphics, and image processing, vol. 34, no. 3, pp. 344-71, 1986. 